\title{
Tratamiento del paro cardiaco en adultos, niños y neonatos con COVID-19. Recomendaciones de la Sociedad Interamericana de Cardiología (SIAC), Asociación Nacional de Cardiólogos de México (ANCAM) y Sociedad Mexicana de Cardiología (SMC)
}

\author{
Treatment of adult, child and newborn cardiac arrest victims with COVID-19. \\ Recommendations from the Interamerican Society of Cardiology / Sociedad \\ Interamericana de Cardiología (SIAC), Mexican National Cardiologists Association / \\ Asociación Nacional de Cardiólogos de México (ANCAM) and Mexican Society of \\ Cardiology / Sociedad Mexicana de Cardiología (SMC)
}

\author{
Humberto Rodríguez-Reyes ${ }^{\star 1}$, Fernando Ortiz-Galván², Martín Ibarrola ${ }^{3}$, Manuel Celaya-Cota ${ }^{4}$, \\ Sergio Dubner ${ }^{6}$, Enrique Asensio-Lafuente ${ }^{6}$, Elaine Núñez Ayala ${ }^{7}$, Pablo Mendoza-Novoa ${ }^{8}$, \\ Luz Ma. M. Muñoz-Gutiérrez ${ }^{9}$, Georgia Sarquella-Brugada ${ }^{10}$, Iván Mendoza ${ }^{11}$ y Manlio F. Márquez ${ }^{12}$
}

${ }^{1}$ Sociedad Cardiovascular y Arritmias, Aguascalientes, México; ${ }^{2}$ Centro Universitario del Sur, Universidad de Guadalajara, Ciudad Guzmán, Jalisco, México; ${ }^{3}$ Centro Cardiovascular BV. Bella Vista. Buenos Aires. Argentina; ${ }^{4}$ Hospital CIMA, Hermosillo, Sonora, México; ${ }^{5}$ Servicio de Electrofisiología y Arritmias, Sanatorio clínica y maternidad de los Arcos, Buenos Aires, Argentina; ${ }^{6}$ División de Medicina Interna, Hospital H, Querétaro, México; ${ }^{7}$ Unidad de Electrofisiología, arritmias y marcapasos, Centro Cardiovascular, Cedimat. Santo Domingo. Rep. Dominicana; ${ }^{8}$ Unidad de arritmias y estimulación cardiaca. Hospital Nacional Dos de Mayo, Lima Perú; ${ }^{9}$ Sociedad Cardiovascular y Arritmias, Aguascalientes, México; ${ }^{10}$ Unidad de Arritmias Pediátricas, Cardiopatías Familiares y muerte Súbita, Hospital Sant Joan de Déu, Barcelona, España; ${ }^{11}$ Cardiología tropical, Universidad Central de Venezuela, Caracas, Venezuela; ${ }^{12}$ Investigación clínica, Instituto Nacional de Cardiología, Ignacio Chávez, ciudad de México, México

\begin{abstract}
Resumen
La pandemia de COVID-19 ha infligido grandes estragos a la población y en especial al personal de salud. Los esfuerzos de reanimación exigen modificaciones potenciales de las guías internacionales existentes de reanimación cardiopulmonar (RCP) debido al elevado índice de contagiosidad del virus SARS-CoV-2. Se considera que hasta 15\% de los casos de COVID-19 tiene una enfermedad grave y $5 \%$ padece un trastorno crítico con una mortalidad promedio del $3 \%$, la cual varía según sean el país y las características de los pacientes. La edad y las comorbilidades como la hipertensión arterial, enfermedad cardiovascular, obesidad y diabetes incrementan la mortalidad hasta $24 \%$. También se ha informado un aumento reciente del número de casos de paro cardíaco extrahospitalario (PCEH). Aunque el paro cardíaco (PC) puede ser efecto de factores diversos en estos pacientes, en la mayoría de los casos se ha demostrado que el origen es respiratorio, con muy pocos casos de causa cardíaca. Se debe considerar la indicación de iniciar o continuar las maniobras de RCP por dos razones fundamentales: la posibilidad de sobrevida de las víctimas, que hasta la fecha se ha registrado muy baja, y el riesgo de contagiar al personal de salud, que es muy alto.
\end{abstract}

Palabras clave: Reanimación cardiopulmonar. Paro cardíaco. COVID-19. Coronavirus. Personal de salud. Recomendaciones.

Correspondencia:

*Humberto Rodríguez-Reyes

E-mail: humbertorodriguezr@ hotmail.com
Disponible en internet: 30-06-2020 Arch Cardiol Mex. 2021;91(Supl):64-73

www.archivoscardiologia.com 1405-9940 / @ 2020 Instituto Nacional de Cardiología Ignacio Chávez. Publicado por Permanyer. Este es un artículo open access bajo la licencia CC BY-NC-ND (http://creativecommons.org/licenses/by-nc-nd/4.0/). 


\begin{abstract}
The COVID-19 pandemic is having a large impact on the general population, but it has taken a specially high toll on healthcare personnel. Resuscitation efforts require potential modifications of the present Cardiopulmonary Resuscitation (CPR) international guidelines because of the transmissibility rate of the new SARS-CoV 2 virus. It has been seen that up to $15 \%$ of COVID-19 patients have a severe disease, $5 \%$ have a critical form of infection and the mean death rate is $3 \%$, although there are significant differences according to the country that reports it and patients' baseline conditions that include age, presence of arterial hypertension, cardiovascular disease, diabetes or obesity. In these high risk subjects, mortality might go up to $24 \%$. There are also reports of a recent increase in out-of-hospital cardiopulmonary arrest (OHCA) victims. Cardiac arrest (CA) in these subjects might be related to many causes, but apparently, that phenomenon is related to respiratory diseases rather than cardiac issues. In this context, the decision to start or continue CPR maneuvers has to be carefully assessed, because of the low survival rate reported so far and the high contagion risk among healthcare personnel.
\end{abstract}

Key words: Cardiopulmonary resuscitation. Cardiac arrest. COVID-19. Coronavirus. Health personnel. Recommendations

\section{Introducción}

La expansión de la pandemia del COVID-19 representa un importante reto para la población y en especial para el personal de salud. Los esfuerzos de reanimación requieren modificar las guías internacionales existentes de reanimación cardiopulmonar (RCP), en virtud del elevado índice de contagiosidad del virus SARSCov- $2^{1,2}$. De acuerdo con datos recientes de la OMS, $40 \%$ de los casos experimenta una enfermedad leve, $40 \%$ puede tiene una afección moderada, $15 \%$ puede desarrollar un trastorno grave y $5 \%$ puede sufrir una enfermedad crítica $^{2}$. La mortalidad cruda varía según sea el país y se observa una mortalidad global del 3\%, que se incrementa con la edad y la presencia de comorbilidades, entre ellas hipertensión arterial, enfermedad cardiovascular, obesidad y diabetes que pueden registrar mortalidad hasta del $24 \%{ }^{3}$. También se ha observado un aumento de casos de paro cardíaco extrahospitalario (PCEH) tanto en Italia ${ }^{4}$ como en EE.UU. ${ }^{5}$ Son múltiples los mecanismos que pueden llevar a un paciente con COVID-19 a sufrir un paro cardíaco (PC): insuficiencia respiratoria progresiva secundaria a síndrome respiratorio agudo grave, choque, tromboembolia pulmonar, daño miocárdico, arritmias ventriculares malignas secundarias a daño miocárdico ${ }^{1,6} 0$ consumo de fármacos que prolongan el intervalo QTc y favorecen taquicardia ventricular (TV) polimórfica o torsades de pointes (TdP), en particular la combinación de hidroxicloroquina y azitromicina ${ }^{7,8}$, además de las comorbilidades que pueden prolongar el QTc, como la hipopotasemia, hipomagnesemia, fiebre, estado inflamatorio y administración de antivirales como el ritonavir'. En la mayor parte de los casos, el PC es de origen respiratorio y en pocos pacientes de causa cardíaca ${ }^{6}$. De manera idónea, es recomendable determinar si existe o no la orden de realizar RCP mediante consentimiento informado del paciente y/o familiares. Con la expansión de la infección en todo el mundo es muy probable que se incremente el número de sujetos con PC por COVID-19. En este artículo se describen las recomendaciones para el personal de salud con el objetivo de proporcionar la mejor atención posible a estos pacientes, así como reducir la exposición y contagio del personal de la salud con todas sus implicaciones; de esta manera es posible reducir el número de casos y la expansión de la infección por COVID-19.

\section{Contagio al personal de salud}

El principal mecanismo de transmisión del COVID-19 es el de persona a persona. Cuando se encuentran en contacto cercano, el virus se propaga en las microgotas de secreción que emite el individuo infectado al hablar, toser o estornudar. Las microgotas pueden alcanzar las mucosas conjuntiva, nasal u oral de la persona sana y de ahí desplazarse a los pulmones ${ }^{1,2}$. Con frecuencia, los sujetos infectados son asintomáticos, lo que contribuye a su transmisión y diseminación y ello eleva el número de casos tanto en la población general como en el personal de salud ${ }^{10,11}$. Este último puede desarrollar una enfermedad más grave por su exposición a una mayor carga viral. En promedio, se considera que más del $10 \%$ de los contagios afecta al personal de salud. El principal factor de riesgo es la falta de equipo de protección personal (EPP) ${ }^{10,12}$. En Latinoamérica, una encuesta reciente de 936 trabajadores del sistema de salud (95.1\% médicos) mostró que sólo contaban con mascarillas $\mathrm{N} 95$ el $56.1 \%$ y mascarilla protectora el $32.6 \%$, lo que revelaba que el personal de salud en 
esta región tiene acceso limitado a EPP, esencial durante la pandemia de COVID-1913.

En China se notificó una incidencia de contagio del personal de salud del $4.4 \%$ al inicio de la epidemia, principalmente por la falta de medidas de protección completas del personal de salud. Hasta la fecha, con la creación de hospitales especiales para atender a este tipo de pacientes, y con la utilización de EPP completo, la incidencia de contagio del personal de salud en China se ha reducido en grado considerable ${ }^{14}$. En los primeros casos de Wuhan se consideró que el $41 \%$ de los contagios se debió a la transmisión intrahospitalaria ${ }^{14,15}$.

En Italia, hasta el 17 de abril, se había comunicado el contagio de 17,000 sujetos del sistema de salud, de los cuales 125 médicos y 34 enfermeras fallecieron ${ }^{16}$. En España se ha informado el 14\% del personal de salud infectado ${ }^{17}$. En Inglaterra se encontró también un $14 \%$ de su personal infectado, $81 \%$ del cual correspondió a los clínicos que trabajan en contacto directo con pacientes, $8 \%$ a los que trabajan en la misma institución sin estar en contacto directo con pacientes y $11 \%$ al personal administrativo del hospital ${ }^{18}$.

En EE. UU., hasta el 9 de abril, se registraron 9,282 casos de contagio en el personal de salud ${ }^{19}$. En Latinoamérica, aunque no se dispone de una cifra exacta, cada vez más se comunican casos de personal de salud infectados; en México, hasta $23 \%$ de los infectados corresponde a clínicos de salud ${ }^{20}$.

\section{Hospitales especiales para tratar a pacientes con COVID-19}

Todos los pacientes con COVID-19 deben tratarse en hospitales diseñados o reconfigurados para el control de infecciones de transmisión aérea, donde se han instituido protocolos estrictos para reducir el riesgo de contagio del personal de salud. Pese a ello, dada la notoria afluencia de pacientes, es probable que en varios países de Latinoamérica ocurra lo mismo que en España, donde los pacientes han tenido que atenderse en cualquier hospital, no sólo en los adaptados con instalaciones especiales.

1. Los pacientes deben colocarse en habitaciones individuales con la puerta cerrada ${ }^{9}$.

2. Todos los pacientes deben someterse a la colocación de máscara o tienda facial para controlar las secreciones que producen la tos, el habla o los estornudos ${ }^{9}$.

3. Es recomendable que todo el personal de salud en contacto directo con estos enfermos utilice equipo de protección personal completo en todo momento. El material para explorar al paciente (p. ej., ecógrafos, electrocardiógrafos, entre otros) que tenga contacto con el enfermo debe protegerse y desinfectarse de forma cuidadosa después de casa uso $0^{1,2,9}$.

4. Para el resto del personal de salud que no esté en contacto directo con el paciente, pero que labore en la misma institución, se recomienda usar equipo de protección personal parcial con al menos cubrebocas, lentes protectores y, cuando sea posible, mascarilla ("caretas"), además de aplicar las medidas de higiene y distanciamiento recomendadas por las autoridades para reducir la posibilidad de contagio ${ }^{1,9}$.

\section{La RCP produce aerosoles}

La RCP exige un esfuerzo de los profesionales de la salud, que trabajan en estrecha proximidad con el paciente y entre sí, por lo que el riesgo de contagio es muy alto ${ }^{1,2,9}$; los esfuerzos de reanimación liberan aerosoles, en especial durante las compresiones torácicas, al ventilar y al tratar de establecer una vía aérea avanzada ${ }^{1}$. Es posible que las mascarillas N95 no proporcionen suficiente protección durante las maniobras de RCP; además, los movimientos corporales intensos y dinámicos de los rescatadores, así como la falta de un ajuste adecuado, favorecen la pérdida de sellado y el riesgo alto de contagio de infecciones que se transmiten por el aire, como el COVID-19 y las epidemias del síndrome respiratorio agudo grave del 2010 y el síndrome respiratorio del Medio Oeste en el 201521. La tabla 1 muestra las recomendaciones generales de $\mathrm{RCP}$ en pacientes con sospecha o confirmación de COVID-19.

\section{Recomendaciones antes de iniciar RCP}

1. Todo el personal que participe en la RCP debe contar con equipo de protección personal completo ${ }^{1,2,9}$ y mascarilla con sellado hermético que no obstruyan la comunicación y la movilización durante la RCP21.

2. El enfermo debe someterse a la colocación de mascarilla o tienda facial para controlar la emisión de aerosoles durante la $\mathrm{RCP}^{1,9}$.

3. Es necesario establecer con anticipación lo adecuado de instituir RCP. Según sean las características y la evolución del paciente, hay que considerar la presencia de comorbilidades, la causa del PC y el ritmo inicial (en caso de asistolia, la posibilidad de respuesta es muy pobre), además de ponderar la RCP y el riesgo de contagiar al personal ${ }^{1,6,9}$. De acuerdo a información reciente en pacientes hospitalizados, se deberá prestar especial atención a los marcadores de inflamación que se han asociado con un peor 
Tabla 1. Recomendaciones generales antes de iniciar las maniobras de RCP en pacientes con sospecha 0 confirmación de COVID-19

1. Reducir la exposición del personal de salud:

a) Utilizar EPP completo antes de iniciar RCP; debe utilizarse mascarilla con sellado hermético que no obstruya la comunicación y la movilización durante la RCP.

b) Limitar al personal durante la RCP.

c) Inicie la evaluación a 2 metros de distancia del paciente.

d) Colocar mascarilla al paciente para limitar la emisión de aerosoles.

e) Al evaluar pulso y respiración, no se acerque para escuchar la respiración.

f) En caso de estar disponibles, considerar el uso de compresores mecánicos para adultos y adolescentes.

g) Notificar a todo el personal de salud que el paciente es portador de COVID-19.

2. Considerar la revisión y organización del equipo de reanimación antes de iniciar las maniobras y asignar las funciones a cada miembro y la secuencia de procedimientos para reducir al mínimo el riesgo de contagiar al personal de salud.

3. Valorar si la reanimación es apropiada:

a) Considerar los factores de riesgo del paciente, la edad, la gravedad de la enfermedad, la causa y el ritmo inicial de PC para establecer lo adecuado o no de iniciar o continuar con RCP.

4. En todo paciente, el equipo médico deberá tratar de evitar en lo posible el PC, si es necesario enfrentarlo, es indispensable estar bien preparados y equipados.

5. Es recomendable tener una discusión con el paciente y su familia, si existe alguna preferencia con relación a los esfuerzos de reanimación, o bajo qué condiciones "no resucitar", orden que será escrita y conservada en el expediente del paciente, siempre y cuando la legislación permita tal posibilidad.

EPP: equipo de protección personal; RCP: reanimación cardiopulmonar; PC: paro cardíaco.

pronóstico tales como dimero-D, proteína C reactiva, ferritina o leucocitosis entre otros.

4. En los casos con PC de origen cardíaco, con ritmo inicial de fibrilación ventricular o taquicardia ventricular (FV/TV), la posibilidad de respuesta a la RCP es mayor; infortunadamente, este comportamiento sólo se ha informado en $5 \%$ de los episodios de PC intrahospitalario 6 .

5. Es recomendable, cuando sea posible, discutir con el paciente y sus familiares si existe alguna preferencia con relación a los esfuerzos de reanimación o bajo que condiciones específicas "No resucitar", orden que puede ser escrita, siempre y cuando la legislación permita tal posibilidad.

\section{Sistema de urgencias médicas}

El despachador de urgencias (911 en varios países) debe identificar síntomas de COVID-19, como fiebre, tos y dificultad para respirar, detectar contactos previos del paciente con otros sujetos con sospecha o confirmación de COVID-19 y notificar al sistema de atención prehospitalaria que la víctima es portadora o tiene alto riesgo de COVID-19. Ante la duda, cualquier paciente debe considerarse portador de COVID-19 hasta no demostrarse lo contrario y, por lo tanto, deben tomarse siempre todas las precauciones. Es importante reconocer que en Italia ${ }^{4}$ y EE.UU. ${ }^{5}$ se han informado más casos de PCEH que los notificados con anterioridad; en Italia se observó un incremento del $58 \%$ de casos, de los cuales una proporción de $77.4 \%$ era sospechosa o había recibido un diagnóstico de COVID-194.

\section{Recomendaciones para familiares y rescatadores no profesionales}

Para todos los rescatadores no profesionales se recomienda sólo realizar compresión torácica; es importante que el rescatador utilice mascarilla para cubrir boca y nariz ${ }^{1}$.

1. Se recomienda usar mascarilla para la víctima y reducir el riesgo de transmisión de la enfermedad ${ }^{1,9}$.

2. En el caso de los niños, deben practicarse compresiones y ventilación; en estos pacientes, la incidencia de paro respiratorio es mayor ${ }^{1}$. Hay que tener en cuenta que la carga viral en los niños es más alta que en los adultos y por tanto son aún mayores las precauciones que deben adoptarse en ellos.

3. Si se dispone de un desfibrilador externo automático deben seguirse sus instrucciones.

\section{Recomendaciones para el personal de salud que atiende un PC extrahospitalario}

De acuerdo con la prevalencia local y la expansión del COVID-19 en la comunidad, se debe considerar que todos los PCEH son efecto de COVID-191,4,5. En las figuras 1 y 2 se muestran los algoritmos de apoyo vital básico en adultos y pediátrico en pacientes con sospecha o confirmación de COVID-19, respectivamente. 
Antes de iniciar RCP utilice EPP completo, reduzca la posibilidad de contagio, limite el personal de salud

난

Víctima no responde, pida ayuda, solicite un DEA

단

Coloque la mascarilla a la víctima, valore el pulso y respiración de forma simultánea por $10 \mathrm{~s}$. No acerque su cara

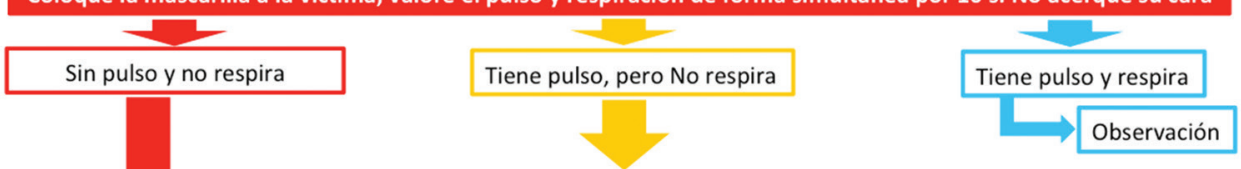

Administre ventilaciones de rescate, con sistema de bolsa-mascarilla con sellado hermético y filtro HEPA, 1 ventilación c/5-6 s (10-12 por min), continúe con ventilación de rescate y revise el pulso; si no tiene pulso, inicie RCP. Sobredosis de opioides: administre naloxona.
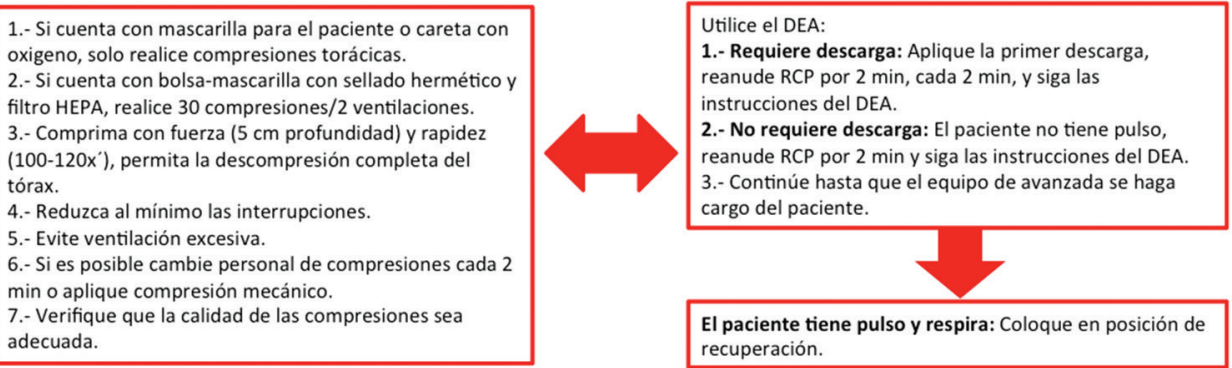

Figura 1. Algoritmo de apoyo vital básico en adultos con sospecha o confirmación de COVID-19.

DEA: desfibrilador externo automático; EPP: equipo de protección personal; filtro HEPA: filtro de aire de alta eficiencia; RCP: reanimación cardiopulmonar.

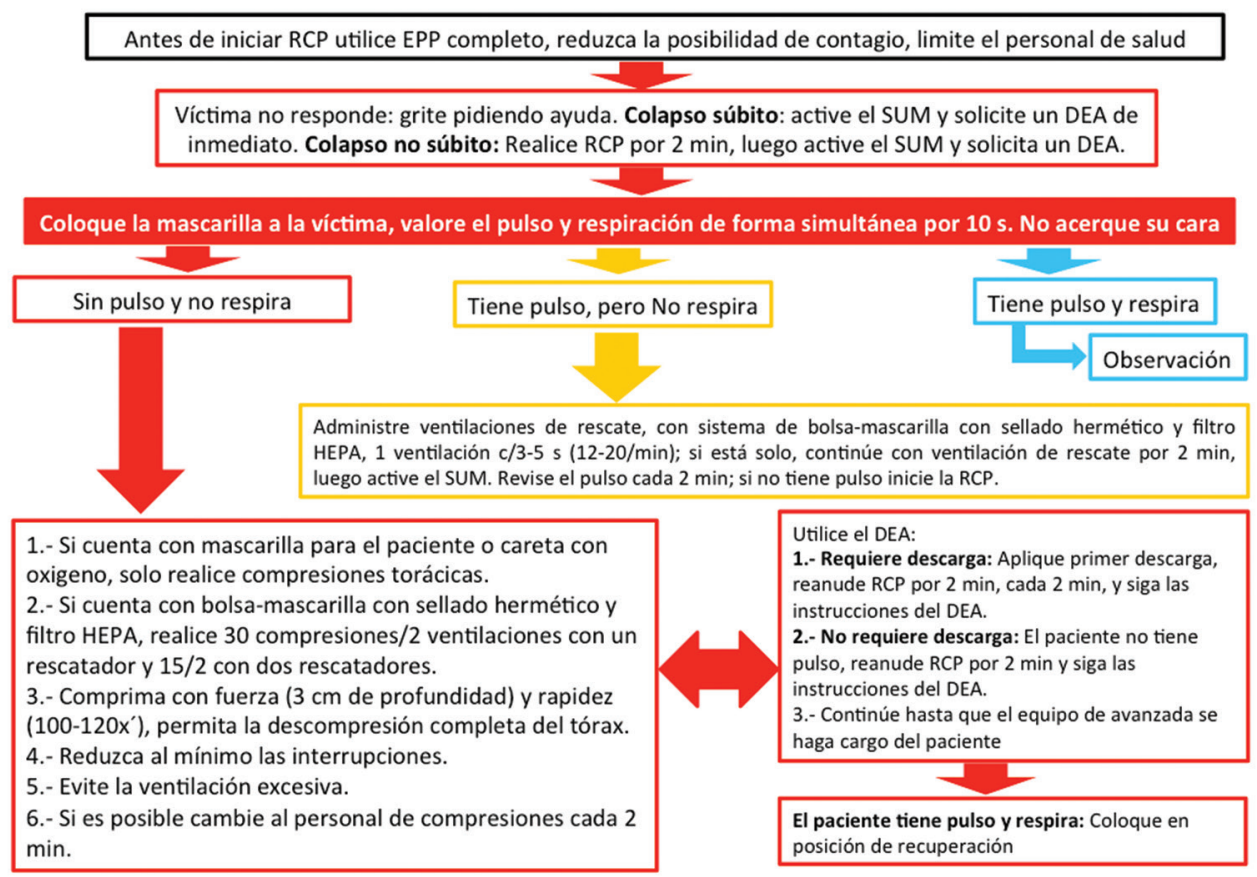

Figura 2. Algoritmo de apoyo vital básico pediátrico con uno o dos rescatadores para pacientes con sospecha 0 confirmación de COVID-19.

EPP: equipo de protección personal; DEA: desfibrilador externo automático; filtro HEPA: filtro de aire de alta eficiencia; RCP: reanimación cardiopulmonar; SUM: sistema de urgencias médicas. 
Antes de iniciar RCP utilice EPP completo, reduzca la posibilidad de contagio, limite el personal de salud

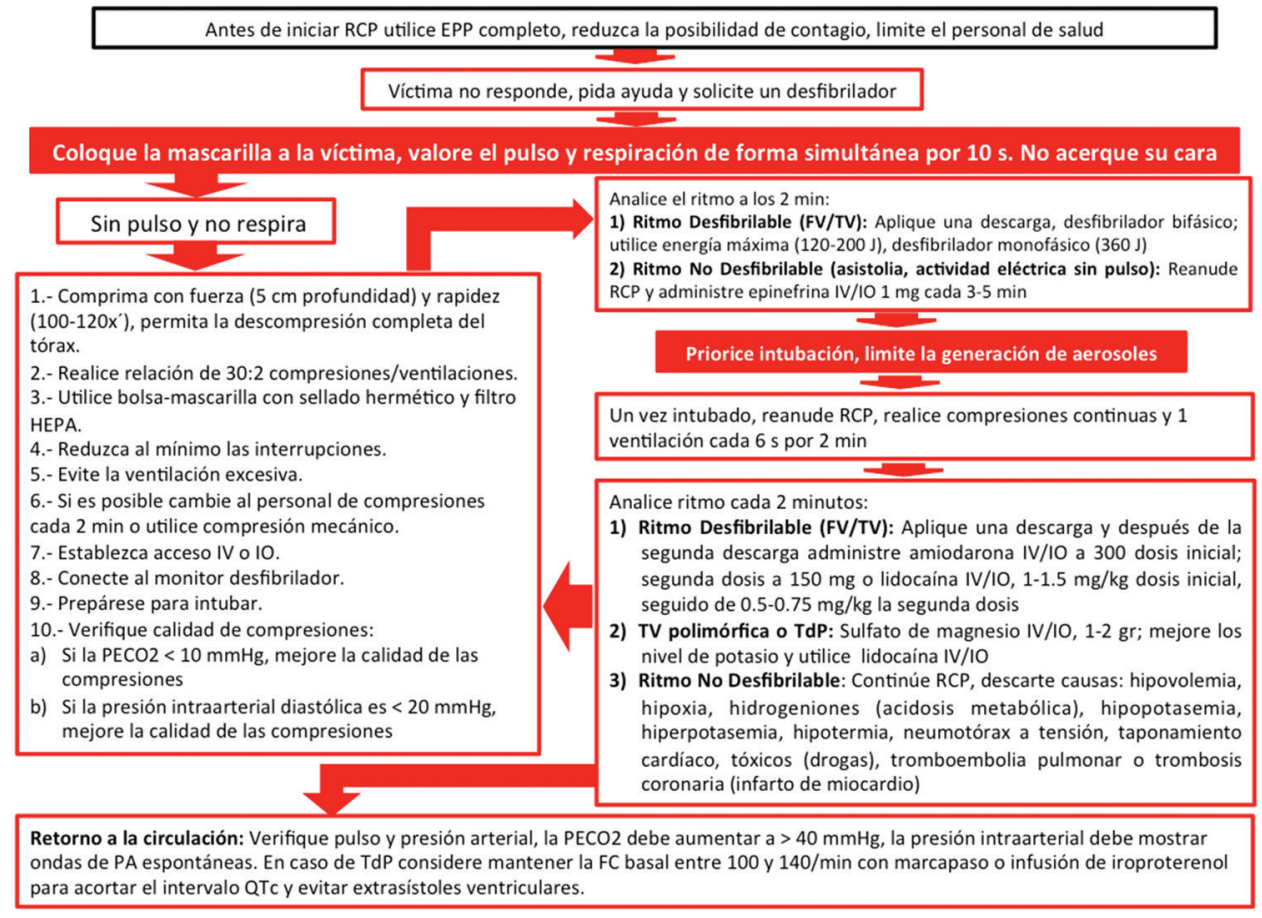

Figura 3. Algoritmo de apoyo vital avanzado para adultos con sospecha o confirmación de COVID-19.

EPP: equipo de protección personal; filtro HEPA: filtro de aire de alta eficiencia; RCP: reanimación cardiopulmonar; IV: intravenoso; IO: intraóseo; $\mathrm{PECO}_{2}$ : presión espirada de dióxido de carbono; FV: fibrilación ventricular; TV: taquicardia ventricular; TdP: torsades de pointes.

1. El personal de atención prehospitalaria debe contar con EPP completo ${ }^{1}$.

2. Una revisión y organización del equipo antes del arribo del paciente, con asignación de las funciones de cada miembro y el flujo de procedimientos, reducen el riesgo de contagio del equipo de salud ${ }^{1}$.

3. Al responder a cualquier paciente con signos o síntomas de infección respiratoria, la evaluación se debe iniciar a una distancia de al menos $2 \mathrm{~m}$ del paciente.

4. El contacto debe ser minimizado en la medida de lo posible, hasta que se coloque una mascarilla facial al paciente.

5. Es probable que todas las personas en el hogar del paciente ya estén expuestas al COVID-19.

6. Debe considerarse la presencia de comorbilidades para determinar la necesidad de iniciar o continuar con la RCP.

7. Sólo deben realizarse compresiones torácicas.

8. El miembro responsable de la vía aérea, colocará un dispositivo supraglótico. No se recomienda realizar intubación, la complejidad del procedimiento aumenta substancialmente el riesgo de contagio. En todo momento debe seguir las recomendaciones de la tabla 2 para reducir la generación de aerosoles.

9. El personal de atención pre-hospitalaria solo debe llevar al enfermo a un hospital preparado para recibir casos con sospecha de COVID-19, y debe notificar a la institución a fin de que puedan tomar las precauciones de control antes de su llegada.

10. Considere utilizar dispositivo de compresión torácica mecánica, para minimizar el riesgo de contagio.

11. Los familiares y otros contactos no deben viajar en el mismo transporte.

12. Si no se logra el retorno a la circulación con las maniobras de RCP, debe considerarse no trasladar al hospital a la víctima por la escasa probabilidad de sobrevida'.

13. Para el retiro del EPP completo se recomienda hacerlo bajo supervisión para evitar contaminación ${ }^{22,23}$.

\section{Recomendaciones para el personal de salud que atiende un PC intrahospitalario}

Estas recomendaciones no se aplican a pacientes sin COVID-19, que deben recibir una RCP común; 
Antes de iniciar RCP utilice EPP completo, reduzca la posibilidad de contagio, limite el personal de salud

Víctima no responde, pida ayuda y solicite un desfibrilador

Coloque la mascarilla a la víctima, valore el pulso y respiración de forma simultánea por $10 \mathrm{~s}$. No acerque su cara

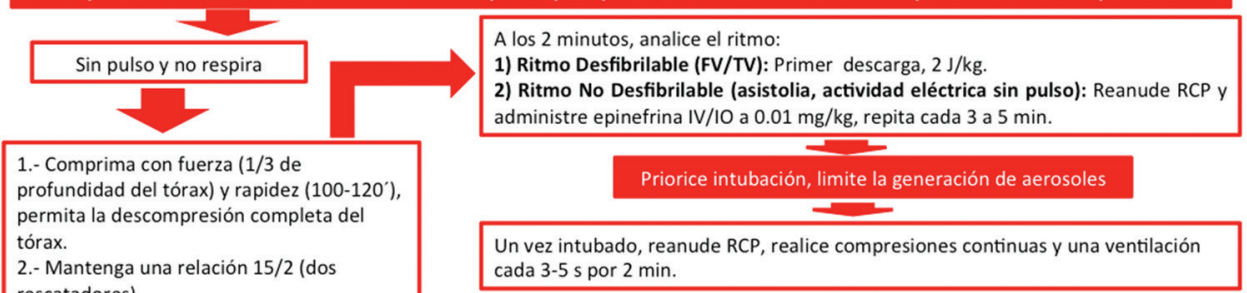

2.- Mantenga una relación $15 / 2$ (dos

3.- Utilice bolsa-mascarilla con sellado

hermético y filtro HEPA.

4.- Reduzca al mínimo las interrupciones.

5.- Evite la ventilación excesiva.

6.- Si es posible cambie personal de

compresiones cada $2 \mathrm{~min}$.

7.- Establezca acceso IV o IO.

8.- Conecte al monitor desfibrilador.

9.- Prepárese para intubar.

Analice ritmo cada 2 minutos:

1) Ritmo Desfibrilable (FV/TV): Segunda descarga a $4 \mathrm{~J} / \mathrm{kg}$, descargas subsecuentes de 4-10 J/kg. Después de la segunda descarga administre amiodarona IV o 10 a $5 \mathrm{mg} / \mathrm{kg}$, en bolo; puede repetir en 2 ocasiones para FV/TV resistentes. Puede usar lidocaina IV o 10 a $1 \mathrm{mg} / \mathrm{kg}$, dosis inicial; a los 15 minutos puede continuar dosis de mantenimiento de $20-50 \mathrm{mg} / \mathrm{kg} / \mathrm{min}$.

2) TV polimórfica o TdP e hipomagnesemia: Sulfato de magnesio IV/10 a $25-50 \mathrm{mg} / \mathrm{kg}$ en 10-20 min, no exceder de $2 \mathrm{gr}$.

3) Ritmo No Desfibrilable: Continúe RCP, descarte causas: hipovolemia, hipoxia, hidrogeniones (acidosis metabólica), hipopotasemia, hiperpotasemia, hipotermia, neumotórax a tensión, taponamiento cardíaco, tóxicos (drogas), tromboembolia pulmonar o trombosis coronaria (infarto de miocardio).

Retorno a la circulación: Verifique pulso y presión arterial; la PECO2 debe aumentar a > $40 \mathrm{mmHg}$, la presión intraarterial debe mostrar ondas de PA espontáneas. En caso de TdP considere mantener la FC basal entre 100 y 140/min con marcapasos o infusión de

iroproterenol para acortar el intervalo QTc y evitar extrasistoles ventriculares.

Figura 4. Algoritmo de apoyo vital avanzado pediátrico en pacientes con sospecha o confirmación de COVID-19

EPP: equipo de protección personal; filtro HEPA: filtro de aire de alta eficiencia; RCP: reanimación cardiopulmonar; IV: intravenoso; I0: intraóseo; $\mathrm{PECO}_{2}$ : presión espirada de dióxido de carbono; FV: fibrilación ventricular; TV: taquicardia ventricular; TdP: torsades de pointes.

Tabla 2. Medidas para la ventilación y la oxigenación con baja generación de aerosoles

1. Utilizar bolsa-mascarilla con sellado hermético y filtro HEPA

2. Intubar en fase temprana y conectar al ventilador mecánico.

3. Para favorecer una intubación exitosa en el primer intento, detener las compresiones y usar videolaringoscopio.

4. Colocar sistema de protección y aislamiento del paciente durante la intubación (Fig. 5).

5. Si la intubación se retrasa o no cuenta por personal con entrenamiento apropiado, considere dispositivo supraglótico (mascarilla laríngea o tubo laríngeo).

6. Minimice la desconexión del circuito cerrado.

Filtro HEPA: filtro de aire de alta eficiencia.

reduzca el número de rescatadores para mantener el distanciamiento y evitar contagio durante la pandemia ${ }^{1}$. En las figuras 3 y 4 se muestran los algoritmos de apoyo vital avanzado en adultos y pediátrico respectivamente, en pacientes con sospecha o confirmación de COVID-19, en ambos se incluye el tratamiento de TV polimórfica o $\mathrm{TdP}^{24,25}$.

1. Se recomienda la vigilancia estrecha del paciente y la identificación de signos de deterioro para reducir la necesidad de intubación de urgencia, que implica alto riesgo para el paciente y el personal de salud.

2. Si el paciente se halla en riesgo alto de PC, hay que considerar su traslado a una unidad de presión negativa, si está disponible, para reducir al mínimo el riesgo de exposición de los reanimadores durante la $\mathrm{RCP}{ }^{1,9}$.

3. Mantener la puerta cerrada para prevenir la contaminación de los espacios adjuntos ${ }^{9}$.

4. Se recomienda que el equipo este compuesto del mínimo de personas necesarias para reducir el riesgo de contagio, cuatro personas se considera un número apropiado.

5. Intubar al paciente al momento del PC y seguir las instrucciones para reducir al mínimo la emisión de aerosoles que se muestran en la tabla 2 . Es recomendable utilizar sistema de protección y aislamiento del paciente durante la intubación y videolaringoscopio para reducir el riesgo de contagio del personal de salud, como se muestra en la figura 5.

6. Ajustar el ventilador a las necesidades de cada paciente. 


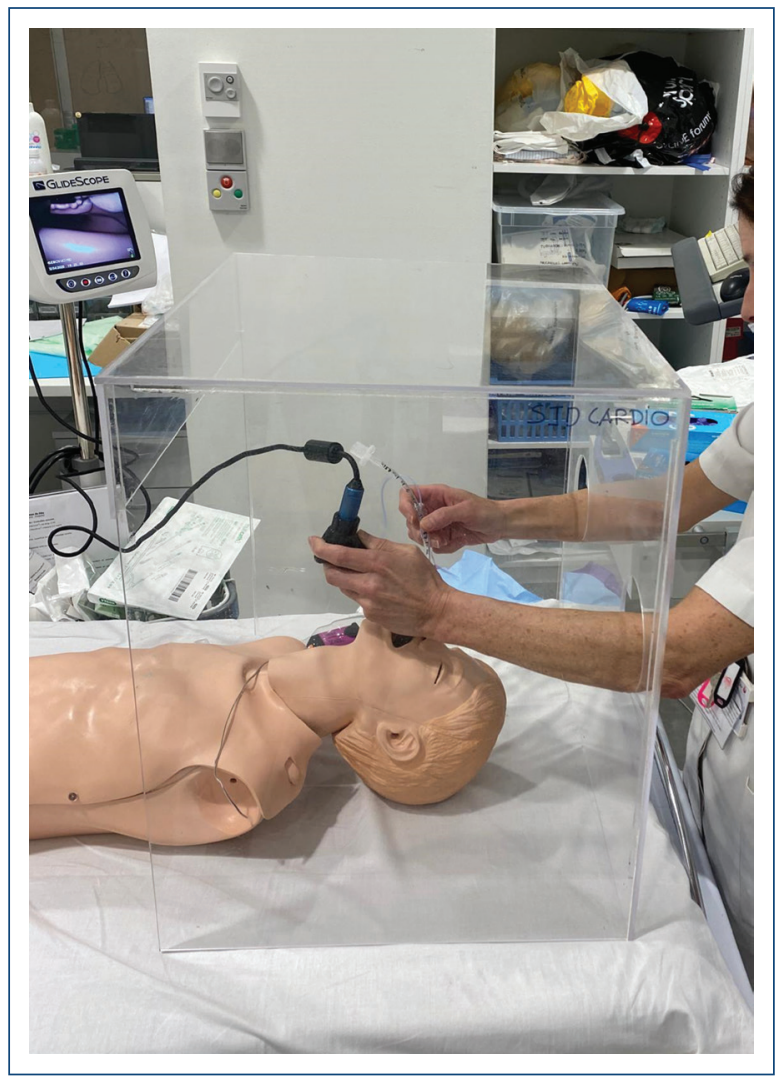

Figura 5. Sistema de protección y aislamiento del paciente durante la RCP e intubación con videolaringoscopio para reducir el riesgo de contagio al personal de salud.

7. En pacientes intubados que presentan un $P C$, incremente la $\mathrm{FiO} 2$ a 1.0, realice compresiones continuas y mantenga el ventilador en modo ventilación con control de presión, limite la presión necesaria para generar una adecuada elevación del tórax $(6 \mathrm{~mL} / \mathrm{kg}$ de peso ideal en adultos, y de 4-6 mL/kg para neonatos), ajuste el trigger en Off. Mantenga una frecuencia de 10/min para adultos y de 30/min para neonatos. Asegure el tubo endotraqueal o la traqueostomía y el circuito de ventilación para prevenir extubación inadvertida. Si se logra el retorno a la circulación, reprograme el ventilador a las condiciones necesarias para el paciente.

8. Si el paciente se encuentra en decúbito prono, cambiarlo al decúbito supino para realizar RCP. Se desconoce si la RCP en decúbito prono es igual de efectiva que en decúbito supino o si puede causar lesión de la columna torácica o medular, pero puede considerarse que, en caso de imposibilidad para cambiar al decúbito supino, se coloquen los parches del desfibrilador en posición anteroposterior del tórax y se valore la práctica de compresiones sobre los cuerpos vertebrales $\mathrm{T} 7 / \mathrm{T} 10^{1}$. Se debe considerar cambiar de posición en caso de compresiones inefectivas en prono de acuerdo con los registros de presión diastólica, que requiera intervención de vía aérea por extubación inadvertida o dificultades para restablecer pronto la circulación.

9. Para el retiro del EPP completo se recomienda hacerlo bajo supervisión para evitar la contaminación ${ }^{22,23}$.

\section{Consideraciones especiales en madre y recién nacido con PC}

Hasta la fecha se ha identificado a pacientes con afectación por COVID-19 con evolución variable, de acuerdo con cada caso (datos no publicados del Hospital Sant Joan de Déu, Barcelona); en consecuencia, se recomienda considerar que la madre y el recién nacido son una fuente de contagio'.

\section{Paro cardíaco neonatal}

Es preferible la administración de epinefrina al neonato por el cordón umbilical para evitar la emisión de aerosoles, que puede ocurrir con aplicaciones endotraqueales ${ }^{1}$. Los procedimientos de intubación deben seguir las mismas recomendaciones para reducir al mínimo la generación de aerosoles según se muestra en la tabla 2.

\section{Paro cardíaco materno}

Los principios del PCR materno no cambian en pacientes con sospecha o confirmación de COVID-191.

1. Los cambios fisiológicos cardiopulmonares durante el embarazo pueden incrementar el riesgo de descompensación aguda en pacientes embarazadas críticamente enfermas con COVID-19.

2. La preparación de cesárea perimortem debe efectuarse en fase temprana en el algoritmo de RCP y se aplica después de 4 minutos de reanimación para posibilitar el montaje del equipo obstétrico y neonatal con EPP completo, incluso si se logra el retorno de la circulación.

\section{Aspectos éticos de la RCP durante COVID-19}

La crisis sanitaria que ha provocado la pandemia de COVID-19 ha obligado al personal y a las instituciones de salud a desarrollar estándares de crisis, que se diferencian radicalmente de los cuidados habituales. 
Para proteger al personal de salud de forma coherente con el marco ético durante esta crisis sanitaria se recomiendan los siguientes puntos ${ }^{26}$. Cuando sea posible, las preferencias de resucitación deberán ser discutidas con el paciente y sus familiares.

1. Iniciar RCP sólo después de que el personal de salud que participe tenga el EPP completo, que debe incluir mascarilla para el personal que efectúe la intubación.

2.El personal de salud sólo debe realizar intervenciones que puedan hacerse de forma segura, por ejemplo desfibrilar y utilizar mascarillas con complemento de oxígeno en el paciente hasta que el equipo apropiado llegue al lugar de la escena.

3. El personal de salud con adecuado entrenamiento y EPP completo no debe negarse a realizar RCP por motivos de seguridad personal, excepto en casos de pacientes con deterioro resistente o irreversible.

4. Los hospitales deben establecer sus estándares de cómo, cuándo y quién realiza la RCP, con la finalidad de ayudar a los médicos a establecer guías y adherirse a los procesos de reanimación, para que éstos sean adecuados y no se prolonguen más de lo apropiado.

\section{Conclusiones}

La pandemia de COVID-19 supone un reto muy grande para toda la población y en especial para el personal de salud debido a su alta contagiosidad, morbilidad y mortalidad, además de que se ha registrado un aumento de los casos de PC extrahospitalario en varios países. Es necesario aplicar cambios en las disposiciones y tratamientos de las víctimas de paro cardíaco. Debe considerarse que las maniobras de RCP producen aerosoles, que elevan el riesgo de contagiar a los clínicos. Todo el personal de salud que asiste a pacientes con sospecha o infección por COVID-19 debe contar con equipo de protección personal completo, antes de iniciar maniobras de RCP. Es indispensable proteger y cuidar al personal que atiende a los pacientes infectados en todos los países, lo cual se ha convertido en un recurso estratégico. Una mayor edad relacionada con comorbilidades, como hipertensión arterial, diabetes mellitus, obesidad, enfermedad cardiovascular y enfermedad pulmonar, representa para los pacientes un alto riesgo de mortalidad. La principal causa de PCR es la insuficiencia respiratoria progresiva y en la mayoría de los casos el ritmo inicial es la asistolia, con pobres posibilidad de respuesta a las maniobras de RCP.
En todos los casos de PC es razonable considerar la edad, las comorbilidades, la gravedad de la enfermedad y el ritmo inicial para determinar lo apropiado de iniciar o continuar las maniobras de RCP; debe realizarse un balance entre la posibilidad de éxito y el riesgo de contagiar a los rescatadores. El personal de salud debe considerar las consecuencias de sufrir enfermedades, desde incapacidad y limitación grave hasta la muerte, lo que limita la atención para los demás enfermos y sus familias. Es necesario que estas recomendaciones se adapten localmente y que se tomen en consideración el sistema de salud, el comportamiento de la enfermedad y la disponibilidad de recursos.

\section{Agradecimientos}

Los autores expresan su agradecimiento a las sociedades de cardiología participantes por el trabajo realizado en colaboración.

\section{Financiamiento}

Los autores no recibieron ningún financiamiento para la elaboración de este trabajo.

\section{Conflicto de intereses}

Lo autores declaran no tener conflicto de intereses.

\section{Responsabilidades éticas}

Protección de personas y animales. Los autores declaran que para esta investigación no se han realizado experimentos en seres humanos ni en animales.

Confidencialidad de los datos. Los autores declaran que en este artículo no aparecen datos de pacientes.

Derecho a la privacidad y consentimiento informado. Los autores declaran que en este artículo no aparecen datos de pacientes.

\section{Bibliografía}

\footnotetext{
1. Interim Guidance for Basic and Advanced Life Support in Adults, Children, and Neonates with Suspected of Confirmed COVID-19: From the Emergency Cardiovascular Care Committe and Get with the Guidances $\circledast$-Resuscitation Adult and Pediatric Task Force of the American Heart Association in Collaboration with the American Academy of Pediatrics, American Association for Respiratory Care, American College of Emergency Physicians, The Society of Critical Care Anesthesiologist, and American Society of Anesthesiologists: Supporting Organizations: American Association of Critical Care Nurses and National EMS Physicians: Edelson, et al. Circulation. 10.1161/CIRCULATIONAHA.120.047463
} 
2. WHO, COVID-19 Strategy update 14 April 2020. https//www.who.int/emergencies/diseases/novel-coronavirus-2019/strategies-plans-and-operations

3. Richardson S, Hirsch JS, Narasimham M, Crawford JM, McGinn T, Davidson $\mathrm{KW}$, et al. Presenting characteristics, comorbidities, and outcomes among 5700 patients hospitalized with COVID-19 in the New York City area. JAMA. doi:10.1001/jama.2020.6775. April 24, 2020.

4. Baldi E, Sechi GM, Mare C, Cnevari F, Brancaglione A. Out-of-hospital cardiac arrest during the Covid-19 outbreack in Italy. NEJM. 2020. DOI:10.1056/NEJMc2010418)

5. D'Ambrosio A. "Probable" COVID-19 death reporting varies by state authorities wrestle with fatal cases that look like COVID but lack test confirmation. MedPage. 2020. https://www.medpagetoday.com/infectiousdisease/covid19/86127?xid=nl_medpageexclusive_2020-04-24\&eu$\mathrm{n}=\mathrm{g} 728008 \mathrm{~d} 0 \mathrm{r} \&$ utm source $=$ Sailthru\&utm medium =email\&utm cam paign=MPTExclusives_042420\&utm term $=\overline{N L}$ Gen_Int_Medpage_Exclusives_Active

6. Shao F, Xu S, Ma X, Xu Z, Lyu J, Ng M, et al. In-hospital cardiac arrest outcomes among patients with COVID-19 pneumonia in Wuham, China. Resuscitation. Doi.org/10.1016//J.resuscitation.2020.04.005

7. Lane J, Weaves J, Kostka K, Duarte-Salles T, Abrahao MT, Alghoul H, et al. Safet of hydroxychloroquine, alone and in combination with azithromycin, in light of rapid wide-spread use for COVID-19: a multinational, network cohort and self-controlled case series study. MedRxiv. 2020. http//doi.org/10.1101/2020.04.08.20054551

8. ESC guidance for the diagnosis and management of CV disease during the COVID-19 Pandemic. Last update on 21 April 2020. Escardio.org/ Education/COVID-19-and-Cardiology/ESC-COVID-19-Guidance

9. CDC, update April 13, 2020. Interim infection prevention and control recommendations for patients with suspected or confirmed coronavirus disease 2019 (COVID-19) in healthcare settings. www.cdc.gov/coronavirus/2019-ncov/hcp/infection-control

10. Wu Z, McGoogan JM. Characteristics of and important lessons from the coronavirus disease 2019 (COVID-19) outbreak in China. Summary of a report of 72314 cases from the chinese center for disease control and prevention. JAMA. 2020;323:13:1239-1242.

11. Rodríguez-Jiménez A, Yared-Meraz A. COVID-19: desde la línea de fuego. https://espanol.medscape.com/verarticulo/5905284_1

12. Christian R. Am I part of the cure or am i part of the disease? Keeping coronavirus out when a doctor comes home. March 18, NEJM. DOI. 10.1056/NEJMMp2004768

13. Delgado D, Pérez W, Sosa A, Ponte-Nefretti C, Mendoza I, Baranchuk A Personal safety during COVID-19 pandemic: realities and perspectives of healthcare workers in Latin America. International Journal of Environ- mental Reserach and Public Health. 2020;17:2798;doi:10.3390/ijerph17082798).

14. Zhan M, Qin Y, Sue X, Zhu Z. Death from Covid-19 of 23 health care workers in China. Editor letter. DOl:10.1056/NEJMc2005696

15. Wang D, Hu B, Hu C, Zhu F, Liu W, Zhang J, et al. Clinical characteristics of 138 hospitalized patients with 2019 novel coronavirus-infected pneumonia in Wuhan, China. JAMA. 2020:323(11):1061-1069. doi:10.1001/jama.2020.1585

16. BusinessDay, 17/04/2020, https://www.businesslive.co.za/bd/world/europe/2020-04-17-about-17000-italian-healthcare-workers-have-covid-19/

17. The New York Times. Virus knocks thousands of health workers out of action in Europe. https://www.nytimes.com/2020/03/24/world/europe/coronarvirus-europe-covid-19.html

18. Hunter E, Price DA, Murphy E, Schim van der Loeff I, Baker KF. First experience of COVID-19 screening of health-care workers in England. JAMA online april 22.2020. doi.org/10.1016/S0140-6736(20)30970-3

19. Burrer SL, de Perio MA, Hughes MM, Kuhar DT, Luckhaupt SE, McDaniel CJ, et al. Characteristics of health care personnel with COVID-19 United States, February 12-April 9, 2020. US Department of Health and Human Services/Center for Disease Control and Prevention. MMWR. 2020;69(15):477-481.

20. Medscape, viernes 24 de abril, 2020. https://espanol.medscape.com/ verarticulo/5905310?src=mkm_latmkt_200424_mscmrk_escoronavirus_

21. Hwang SY, Yoon H, Yoon A, Kim T, Lee G, Jung KY, et al. N95 filter facepiece respirators do not reliably afford respiratory protection during chest compression: a simulation study. American Journal of Emergency Medicine. 2020:38:12-17.

22. CDC, severe acute respiratory syndrome. Sequence for donning and removing personal protective equipment (PPE). https://www.cdc.gov/ sars/downloads/ppeposter1322.pdf

23. OMS pasos para colocar y retirar equipo de protección personal. https:// www.who.int/csr/resources/publications/ebola/ppe-steps/es/

24. Al-Khatib SM, Stevenson WG, Ackerman MJ, Bryant WJ, Callans DJ, Curtis AB, et al. 2017 AHA/ACC/HRS Guideline for management of patients with ventricular arrhytmias and the prevention of sudden cardiac death. J Am Coll Cardiol. 2018;72:e91-220. https://doi.org/10.1016/j. jacc.2017.10.054

25. Thomas SH, Behr ER. Pharmacological treatment of acquired QT prolongation and torsades de pointes. $\mathrm{Br} J$ Clin Pharmacol. 2016;81(3):420ロ427. doi:10.1111/bcp.12726

26. Kramer DB, Lo B, Dickert NW. CPR in the Covid-19 era - an ethical framework [published online ahead of print, 2020 May ${ }^{6}$. N Engl J Med. 2020;10.1056/NEJMp2010758. doi:10.1056/NEJMp2010758. 